\title{
BMJ Open Body composition and chemotherapy toxicity in women with early breast cancer (CANDO-3): protocol for an observational cohort study
}

\author{
Kesta Durkin (D) , ${ }^{1}$ Adam Heetun, ${ }^{1}$ Sean Ewings (D) , ${ }^{2}$ Richard Munday, ${ }^{3}$ \\ Stephen A Wootton, ${ }^{4}$ Lesley Turner, ${ }^{5}$ Ellen R Copson, ${ }^{1}$ CANDO-3 Steering Group, ${ }^{6}$ \\ Ramsey I Cutress ${ }^{1}$
}

To cite: Durkin K, Heetun A, Ewings S, et al. Body composition and chemotherapy toxicity in women with early breast cancer (CANDO-3): protocol for an observational cohort study. BMJ Open 2022;12:e054412. doi:10.1136/ bmjopen-2021-054412

- Prepublication history for this paper is available online. To view these files, please visit the journal online (http://dx.doi org/10.1136/bmjopen-2021 054412).

Received 10 June 2021 Accepted 26 January 2022
Check for updates

(C) Author(s) (or their employer(s)) 2022. Re-use permitted under CC BY-NC. No commercial re-use. See rights and permissions. Published by BMJ.

For numbered affiliations see end of article.

Correspondence to

Dr Kesta Durkin;

K.L.Durkin@soton.ac.uk

\section{ABSTRACT}

Introduction Systemic anticancer therapy is given to selected patients with early breast cancer (EBC) before or after surgery with the aim of eradicating micrometastatic spread and reducing the risk of cancer recurrence. Chemotherapy treatment is most effective when patients receive the optimum dose, on time and without delays or reductions in their treatment doses. Most chemotherapy drugs are dosed according to body surface area calculated from a patient's height and weight. These calculations were however designed based on data from normal weight patients. This has resulted in uncertainty as to the optimal dosing for patients with different amounts of blood, muscle and fatty tissue (body composition). This study uses segmental bioelectrical impedance analysis (using the Seca mBCA 515) to determine whether differences in the measures of resistance and reactance, and derived estimates of body composition, are predictive of chemotherapy toxicity in the treatment of EBC.

Methods and analysis A prospective observational cohort study of women with EBC in whom adjuvant or neoadjuvant chemotherapy is planned. A total of 300 participants will be recruited across nine UK hospital sites. The primary outcome is to determine if higher fat mass index is associated with increased National Cancer Institute Common Terminology Criteria for Adverse Events v5.0 grade 3 (or higher) chemotherapy toxicity.

Ethics and dissemination This study has received ethical approval from the South Central Hampshire B Research Ethics Committee, England (19/SC/0596: IRAS: 263666). The chief investigator and coinvestigators will be responsible for publication of the study findings in a peerreviewed journal, on behalf of all collaborators.

Trial registration number ISRCTN79577461.

\section{INTRODUCTION}

Obesity is an established risk factor for development of postmenopausal breast cancer and a meta-analysis of over 100 case-control studies showed a $13 \%$ increased risk per 5 $\mathrm{kg} / \mathrm{m}^{2}{ }^{1}{ }^{1}$ Factors implicated include a direct effect of adipose tissue on levels of circulating hormones, such as insulin, insulin-like
Strengths and limitations of this study

- Prospective study that explores the separate and combined relationships between fat mass (FM), fatfree mass (FFM) and skeletal muscle mass together with impedance measures on chemotherapy toxicity.

- Observational study designed to assess associations but not causation.

- Bioelectrical impedance analysis (BIA) is a convenient, cost-effective and quick method for looking at changes in raw values of resistance, reactance and phase angle, and derived estimates of FFM and FM.

- Measurements can be performed on the same days as routine clinic appointments/prechemotherapy blood tests, meaning no extra visits for participants.

- BIA is non-invasive, does not involve radiation and can be measured on all patients with early breast cancer.

growth factors and oestrogens, creating an environment that encourages carcinogenesis and discourages apoptosis, as well as stimulation of the body's inflammatory response. ${ }^{2}$ In contrast, most studies in premenopausal women have concluded that obesity does not increase the risk of development of breast cancer; the mechanism for these phenomena remains unclear. ${ }^{34}$

A high body mass index (BMI) is also associated with reduced overall survival in patients with breast cancer and this effect does not appear to be limited to postmenopausal women. A meta-analysis of 43 studies enrolling patients diagnosed between 1963 and 2005 showed obesity associated with poorer overall survival (HR 1.33) and breast cancer-specific survival (HR 1.33) with a more marked effect in premenopausal women (HR 1.47). ${ }^{5}$ Ewertz et al reported a significant increase in risk of developing distant metastases for patients with a BMI of $25 \mathrm{~kg} / \mathrm{m}^{2}$ or 
greater as compared with patients with a BMI less than 25 $\mathrm{kg} / \mathrm{m}^{2}$ (46\% vs $\left.42 \%\right) .{ }^{6}$ The Southampton-based Prospective Study of Sporadic and Hereditary Breast Cancer in Young Women (POSH) confirms that, in women aged 40 years and under at diagnosis, obese patients have significantly lower 8-year overall survival than healthy weight patients $(58.6 \%$ vs $73.3 \%$, p $<0.001){ }^{7}$

The underlying mechanism is likely to be multifactorial. Patients with a high BMI tend to present later with larger tumours due to their body habitus. ${ }^{6}$ Some studies have also indicated an increased incidence of biologically adverse features, including oestrogen receptor negative tumours, in obese patients. ${ }^{8}$ It is also possible that patients with a high BMI receive less effective treatment for early breast cancer (EBC). Surgical complications occur with a higher incidence in obese patients, potentially delaying systemic therapies. ${ }^{9}$ Ewertz et al reported that both chemotherapy and endocrine therapy seemed to be less effective in patients with BMI of $30 \mathrm{~kg} / \mathrm{m}^{2}$ or greater. $^{6}$

Chemotherapy dose reductions are more common in those with obesity, ${ }^{10}$ and while a meta-analysis showed no evidence for increased toxicity in obesity it was unclear if there was confounding by poorly specified dose capping and use of growth factors. ${ }^{11}$ In a more recent study, higher rates of toxicity were seen in obese compared with healthy weight patients when receiving dose-dense chemotherapy. ${ }^{12} \mathrm{~A}$ review by the American Society of Clinical Oncology (ASCO) concluded that up to $40 \%$ of obese patients with cancer currently receive limited chemotherapy doses that are not based on actual weight, and hypothesised that this may explain the higher cancer mortality rates observed in overweight and obese individuals. ${ }^{13}$ The ASCO review found no strong evidence that short or long-term toxicity is increased among obese women receiving full-weight-based chemotherapy doses and has therefore recommended the use of full-weightbased cytotoxic chemotherapy doses in obese patients in the curative and adjuvant setting. However, data were limited and generally limited to first cycle doses and/or haematological toxicity. Their panel therefore recommended further research to guide appropriate dosing of obese patients with cancer. Our own analysis of chemotherapy prescription records for the 77 POSH participants who were treated with adjuvant chemotherapy at the Southampton Oncology Centre indicates that obese patients did not routinely receive capped chemotherapy doses but were significantly more likely to receive one or more dose delays due to toxicity $(33.3 \%$ vs $5.9 \%$, $\mathrm{p}=0.0068$ ) than normal weight patients. ${ }^{7}$

Most chemotherapy drugs are dosed according to predicted body surface area (BSA). Patients with a similar BSA or BMI may have wide variations in amount and distribution of adipose tissue and skeletal muscle (body composition). While there may be an association between BMI and both fat mass (FM) and fat-free mass (FFM), there is at least a twofold variability in both FM and FFM for any given BMI or BSA over the range of values usually seen in women with breast cancer. ${ }^{14}$
There is accumulating evidence that differences in body composition are important determinants of chemotherapy outcomes. Using estimates of body composition derived from single-slice CT scans obtained in routine clinical care, A systematic review by Kazemi-Bajestani and colleagues indicated that a lack of lean tissue and skeletal muscle mass (SMM) is associated with chemotherapy toxicity across a number of differing types of cancers most typically associated with a low BMI, wasting or cachexia, ${ }^{15}$ and a meta-analysis of two studies suggested that sarcopenia predicts chemotoxicity in patients with metastatic breast cancer. ${ }^{16}$ It has been proposed that lean body mass may, in some instances, be a better predictor of drug dosage possibly since it is a predictor of volume of distribution for some drugs and correlates with liver volume and blood flow. ${ }^{17}$ In contrast to the relatively large number of studies in wasting conditions, few studies have looked at the effect of body composition on chemotherapy tolerance in women with EBC. Two small retrospective series using body composition data derived from CT images indicate that sarcopenia (low muscle mass) is associated with increased chemotherapy toxicity, regardless of overall BMI. ${ }^{18}{ }^{19}$ Del Fabbro et al recently reported that overweight patients had a lower pCR rate to neoadjuvant chemotherapy for $\mathrm{EBC}$, and found that in normal BMI patients with sarcopenia the pCR rate was higher. ${ }^{20}$ While the lack of lean tissue appears to be an important determinant of toxicity, this study also demonstrated that patients with sarcopenia who were overweight or obese had an inferior outcome than normal weight patients with sarcopenia.

While much work has focused on lack of lean mass being associated with treatment-related toxicities, relatively little attention has been directed towards determining the association with increased FM. van den Berg and colleagues assessed body composition by dual-energy X-ray absorptiometry (DXA) scan in women with EBC receiving chemotherapy and observed a higher absolute and higher relative FM was associated with an increased risk of toxicity-induced modifications of treatment, but not absolute lean mass, with the greatest risk observed in those with high fat and low lean mass. ${ }^{21}$ Prado and colleagues $^{22}$ investigated the associations between body composition derived from single-slice CT scans and chemotherapy toxicity in advanced ovarian cancer. They reported that a clear association between both FM and lean body mass (primarily driven by FM) emerged in explaining toxicity. This association was only noted in individuals with excess body weight, with a lower ratio predicting higher exposure and risk for toxicity. Using single-slice CT image analysis, Shachar and colleagues found that low skeletal muscle area and muscle attenuation was associated with increased chemotherapy treatment-related toxicities but found no association with subcutaneous or visceral adipose tissue area. ${ }^{23}$

These observations highlight the need to better understand the impact of differences in body composition on chemotherapy prescribing and toxicity in women with 
EBC. What remains unclear is whether the increased chemotoxicity associated with higher BMI in women with breast cancer is associated with a lack of FFM (sarcopenia), greater FM (excess adiposity) or some interaction between the two. In contrast to the work of Baracos' group, it is not possible to assess body composition from singleslice CT images in most women with EBC as the majority of patients would not undergo staging investigations for distant metastatic disease in the absence of symptoms, ${ }^{24}$ and so these images would not be routinely available. Moreover, the clinical utility of determining body composition from single-slice CT images has been challenged after poor accuracy and precision was demonstrated in comparison to that found with DXA. ${ }^{25}$ While it is possible to use DXA to derive estimates of body composition, such measures are not readily used in oncology patients in routine clinical practice and it cannot be used repeatedly over time to follow the changes in body composition due to constraints of radiation exposure. This is possible with segmental bioelectrical impedance analysis (sBIA), which will be used in this study to determine whether differences in the measures of resistance and reactance, and derived estimates of body composition, are predictive of chemotherapy toxicity in the treatment of breast cancer.

\section{RATIONALE}

All of the methods used to measure body composition in patients with and receiving treatment for breast cancer have technical and practical limitations, most notably associated with the assumptions that underlie the prediction of body composition, or their accessibility for application in routine clinical care. ${ }^{6}$ BIA is readily accessible and measures the biophysical properties of the body in terms of resistance and reactance from which estimates of total body water, either at the whole body level or with sBIA at the level of individual limbs. ${ }^{78}$ Resistance is largely determined by the distance the current has to travel (eg, hence the practice of expressing values adjusted for height) and the ability of the current to pass through the tissue which is largely dependent on water content. Reactance is directly measured and principally reflects the capacitance of tissue and the integrity of the cells through which the current flows. Phase angle is a summative statement that reflects the relationship between resistance and reactance at different frequencies. BIA is not a direct assessment of body composition and its utility as an indicator of body composition relies on the relationship between measures of resistance and reactance and total body water and the generation of regression models against other methods of body composition, preferably multicomponent modelling and/or whole body MRI. These regression equations are both device and population specific and dependent on several key assumptions including homogenous composition, fixed cross-sectional area, consistent distribution of current density and constant tissue hydration. The raw measured values of resistance and reactance and the derived parameter of phase angle are not affected by the factors that affect or assumptions used in the estimation of body composition and have both excellent accuracy and precision. Where device-specific reference ranges of healthy individuals are available, both the measured impedance values, phase angle and derived estimates of body composition can be standardised and expressed as SD scores (or Z scores) adjusted for age, gender and BMI.

The 2021 ASCO guideline update on appropriate systemic therapy dosing for obese adult patients with cancer ${ }^{26}$ recommends prospective studies to 'explore the role of body composition in predicting dose limiting toxicities'. This study design directly addresses that recommendation and we have previously demonstrated the feasibility and acceptability of incorporation of sBIA measurements into routine clinical care using a phase-sensitive sBIA instrument (Seca mBCA 515) in a pilot study (Cancer ; Nutrition and Dietary Origins-2 (CANDO-2)) which provided preliminary data on the correlation between CT and BIA, and on changes in impedance measures during systemic chemotherapy. ${ }^{27}$ This particular device has been validated extensively and shown to yield directly comparable measures of body composition obtained by both four compartment models and whole body MRI in healthy cohorts. ${ }^{28}{ }^{29}$ The central proposition that underlies this programme of work is that it is possible to use sBIA to mark differences between women with EBC in their resilience to chemotherapy. This may be related either to differences in relative chemotherapy dosing arising from differing amounts and proportions of lean and fat tissue and/or differences in physiological and metabolic state relating to both structure (amounts and proportions of lean and fat) and the quality of tissue (reflected in the bioelectrical properties of the body). The study is designed with broad inclusion criteria, minimal exclusions and is multicentre in nature with a view to minimising bias by recruitment of a representative population of patients with EBC receiving chemotherapy.

\section{HYPOTHESIS}

Differences in bioelectrical impedance measures of resistance, reactance and phase angle and/or derived estimates of elevated fat mass index (FMi) and low-fat FFM index (FFMi) are individually and jointly predictive of chemotherapy toxicity.

\section{OBJECTIVES}

Primary objective

- To determine if higher FMi is associated with increased Common Terminology Criteria for Adverse Events (CTCAE, National Cancer Institute; v5.0) grade 3 or higher chemotherapy toxicity ${ }^{30}$ in women receiving neoadjuvant/adjuvant chemotherapy for EBC.

\section{Secondary objectives}

- To determine the prognostic potential of sBIAderived body composition measures including FFMi in predicting grade 3 or higher chemotherapy toxicity. 
- To determine whether patients with EBC with different body composition patterns: (1) are planned to receive the same neoadjuvant/adjuvant chemotherapy dose intensity, and (2) receive the planned neoadjuvant/ adjuvant chemotherapy dose intensity.

- To determine the relationship between sBIAmeasured physical properties, for example, phase angle and reactance and grade 3 or higher chemotherapy toxicity.

- To determine in exploratory analyses the association of body composition measures across all grades of chemotherapy toxicities.

- To determine the concurrent validity of sBIA, with (where available) DXA and where performed as part of routine clinical care, CT approaches to determining body composition at baseline in these women.

\section{SUMMARY OF METHODS AND ANALYSIS Study design}

A multicentre observational (non-interventional) investigator-led academic prospective cohort study. The University Hospital Southampton NHS Foundation Trust is the study sponsor and a recruiting site. The cohort will consist of a minimum of 300 women referred to breast oncology clinics with a diagnosis of early invasive breast cancer and recommended to receive neoadjuvant/adjuvant chemotherapy. The study opened on 16 March 2020 and is currently planned to run until 30 June 2023.

The study's inclusion and exclusion criteria are listed in table 1.

Patients will be recruited over an 18-month period from the nine UK hospital sites (The Christie Hospital, Manchester; Southampton General Hospital, Southampton; Churchill Hospital, Oxford; Royal Hampshire County Hospital, Winchester; Royal Devon and Exeter Hospital, Exeter; Salisbury District Hospital, Salisbury;
Royal Cornwall Hospital, Truro; St James' Hospital, Leeds; and Queen Alexandra Hospital, Portsmouth). The patients will be recruited to the study from Breast Cancer Clinics and Breast Oncology Clinics, screened for eligibility through multidisciplinary meetings and introduced to the study when recommended to receive chemotherapy. They will be introduced to the study at their clinic appointment and if interested will be handed a patient information sheet. After a minimum of 24 hours to study the patient information sheet, they will be contacted to confirm whether they want to take part. Patients will provide informed consent in the form of a paper consent form on the day of the first study visit (V1).

Weight, resistance, reactance and phase angle together with derived estimates of FM, FFM and SMM will be obtained using the Seca mBCA 515 and its associated Seca 115 software at all study centres at each study visit, together with measures of maximal voluntary contraction using a hydraulic hand dynamometer. Impedance and body composition variables will be expressed as absolute values, adjusted for height and standardised as $\mathrm{Z}$ scores against an age, gender and BMI device-specific reference population. ${ }^{31}$ Participants will also be asked to complete the quality of life and lifestyle (European Organisation for the Research and Treatment of Cancer Quality of Life Questionnaire EORTC QLQ-C30 and EORTC QLQ-BR23 (breast cancer)), ${ }^{32}$ physical activity (International Physical Activity Questionnaire-Short Form (IPAQ-SF) $)^{33}$ and appetite and eating habit (Council on Nutrition Appetite Questionnaire (CNAQ)) questionnaires ${ }^{34}$ at baseline and at three other study visits, and alcohol use (Alcohol Use Disorders Identification Test-Consumption (AUDIT-C) $)^{35}$ questionnaires at baseline only. Routine clinical data will be obtained from electronic patient records and include a height measurement as recorded at the first clinic visit,

Table 1 Inclusion and exclusion criteria for the Cancer; Nutrition and Dietary Origins-3 (CANDO-3) study

\section{Inclusion criteria}

Early invasive breast carcinoma.

Stage I-III disease.

- Tumour grade, ER (oestrogen receptor) and HER2 (human epidermal growth factor receptor 2) status available.

- Clinical or pathological tumour size and lymph node status available.

- Neoadjuvant or adjuvant systemic chemotherapy recommended by local breast multidisciplinary meeting.

- No prior systemic anticancer treatment within the past 10 years.

- No evidence of distant metastatic disease.

- Patient agrees to receive neoadjuvant/adjuvant chemotherapy.

- Planned to receive greater than $4 \times 21$ day cycles of anthracycline or taxane-based combination chemotherapy. Twenty-one-day combination regimens including weekly treatments are allowed, for example: (1) carboplatin D1/paclitaxel D1, D8, D15; (2) EC (epirubicin and cyclophosphamide)-weekly paclitaxel.

- Aged $\geq 18$ and $<80$ years.

- Female.

- Able to complete written records in English.

\section{Exclusion criteria}

- Previous invasive malignancy (with the exception of non-melanomatous skin cancer) within the past 10 years.

- Any other medical conditions preventing physical participation in the study procedures.

- Patients receiving only single agent or weekly neoadjuvant/adjuvant chemotherapy regimens, for example, weekly paclitaxel with trastuzumab.

- Patients with existing conditions known to affect body water or cause oedema or muscle conditions that may affect muscle mass such as muscular dystrophies.

- Pregnancy.

- Pacemakers 
blood test results, patient and tumour characteristics, comorbidities, cancer treatments and progress through treatment pathway. Chemotherapy toxicity will be recorded at each clinic visit according to the CTCAE v5.0 (National Cancer Institute), together with full details of chemotherapy, including any dose adjustments, delays or changes to the treatment regimen, to determine chemotherapy delivery (of planned dose density).

Staging and radiology planning CT scan images obtained in routine clinical care, where available prior to visit 1 , will be accessed directly from the hospital picture archiving and communication system (PACS) department and link anonymised using a unique patient study identification number. Images from external centres will be sent using standard NHS image transfer processes between hospitals to the Southampton PACS department. SliceOmatic (V.5.0 Rev-7, TomoVision, Magog, Canada) software will be used to determine tissue cross-sectional areas and attenuation according to the modified Alberta Protocol (Alberta Protocol, TomoVision).

In an optional substudy, patients in Southampton will be invited to undertake further body composition analysis by DXA to determine FM, FFM and appendicular FFM, and to donate one extra $10 \mathrm{~mL}$ blood sample which will be biobanked for future analysis and research.

The study schedule is shown in table 2 .

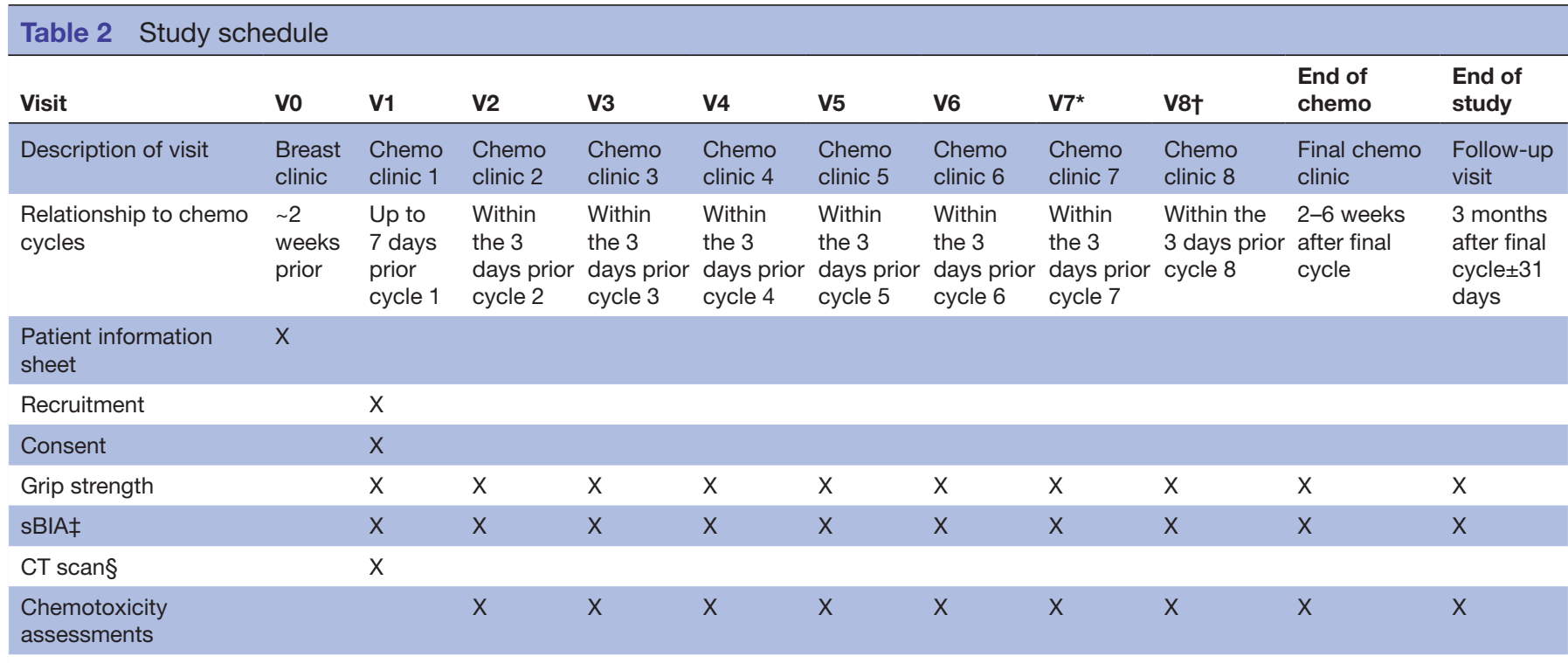

\begin{tabular}{|c|c|c|c|c|}
\hline \multicolumn{5}{|l|}{ Questionnaires } \\
\hline $\begin{array}{l}\text { EORTC-QLQ } \\
\text { (European } \\
\text { Organisation for } \\
\text { the Research and } \\
\text { Treatment of Cancer } \\
\text { Quality of Life } \\
\text { Questionnaire)-C30 }\end{array}$ & $x$ & $x$ & $x$ & $x$ \\
\hline $\begin{array}{l}\text { EORTC QLQ-BR23 } \\
\text { (breast cancer) }\end{array}$ & $x$ & $x$ & $\mathrm{X}$ & $\mathrm{x}$ \\
\hline $\begin{array}{l}\text { IPAQ-SF } \\
\text { (International } \\
\text { Physical Activity } \\
\text { Questionnaire-Short } \\
\text { Form }\end{array}$ & $X$ & $x$ & $x$ & $\mathrm{x}$ \\
\hline $\begin{array}{l}\text { CNAQ (Council on } \\
\text { Nutrition Appetite } \\
\text { Questionnaire) }\end{array}$ & $x$ & $\mathrm{X}$ & $\mathrm{X}$ & $\mathrm{x}$ \\
\hline
\end{tabular}

*Only required if $>6$ cycles of chemotherapy planned.

†Only required if $>7$ cycles of chemotherapy planned. Please name visits to correspond to the chemotherapy cycle that follows that visit (ie, visit 1 =prechemotherapy cycle 1; visit $2=$ prechemotherapy cycle 2 , etc).

¥Weight, impedance and derived estimates of body composition by sBIA.

§Baseline staging CT scan would usually be at some point prior to chemotherapy, although timing can be variable but on entry to the study the patient will consent to use of the data for body composition analysis.

sBIA, segmental bioelectrical impedance analysis. 


\section{Sample size}

A University of Southampton, University Hospital Southampton NHS Foundation Trust-sponsored pilot study (CANDO-2) found that study mean FM corrected for differences in height (FMi) was 11.30 (SD 4.93) kg/ $\mathrm{m}^{2}$ in patients without toxicity (grade 3 or above) and 13.31 (difference of 2.01) in patients with a grade 3 or above toxicity. ${ }^{22}$ Based on simulations, a difference of this magnitude would lead to an area under the curve (AUC; as a measure of discrimination) of over 0.6 based on FMi alone, suggesting at least modest discriminative ability of FMi for predicting toxicity. A sample of size 256 would ensure $90 \%$ power for a two-group comparison on FMi (with alpha of 5\%), and inflating this to 300 maintains this level of power when the groups are unbalanced in size, up to 30-70 in favour of non-toxicity (where such a split has been in related literature) ${ }^{23}$ As such, a statistically significant result would be an indication that FMi shows predictive ability that would be worth exploring in further research since this magnitude of FMi difference is likely to be clinically significant if proven.

\section{Statistical analysis plan}

For the primary objective analyses, descriptive statistics will be used to describe the FMi in patients with versus without a grade 3 or above chemotherapy toxicity, and a formal comparison carried out using a t-test in order to determine if the mean FMi is different between groups. Additional prespecified primary objective analyses will include fitting a logistic regression model to analyse the FMi data according to grade 3 or above toxicity outcome, adjusting for potential confounding variables, for example, BMI. AUCs will also be produced to determine the predictive ability of FMi. Unadjusted and adjusted relative risks and corresponding $95 \%$ CIs, derived using Poisson regression models, will also be reported for associations between FMi and grade 3 or above toxicity outcome. Trial measurements will be made at routine clinic visits and there is no ongoing follow-up so it is anticipated that there will be minimal missing data.

\section{Data management plan, data collection and ALEA eCRF}

A data management plan for the CANDO-3 study has been written and approved. Linked anonymised data will be kept on a secure and trusted validated ALEA eCRF (FormsVision, Netherlands), certified by registered auditors to be in compliance with regulations such as the Food and Drug Administration's CFR (Code of Federal Regulations) 21 Part 11 and ICH GCP (International Conference for Harmonisation Good Clinical Practice). FormsVision supports University Hospital Southampton NHS Foundation Trust and many other organisations, including the University of Cambridge, Leicester and Bristol, and the WHO, with clinical trials data management.

All anonymised clinical, prescribing and toxicity data, and results of the DXA and CT image analysis will be recorded on paper Case Report Forms (CFR's) and centrally entered into a common data set in accordance with local GCP and data protection governance requirements. The BIA data will be recorded electronically at source and uploaded electronically into the common data set.

Data will be cleaned and data set secured under guidance and management by the University of Southampton Clinical Informatics Research Unit Data Management team prior to analyses. The data set will then be analysed in accordance with the Statistical Analysis Plan under the direction of statistical analysis team, Principal Investigator and coapplicants.

\section{PATIENT AND PUBLIC INVOLVEMENT}

A patient advocate (LT) is involved in the design, management and undertaking of this study as a member of the study steering group.

This protocol has been developed with advice from the patient advocate who has extensive experience both with Independent Cancer Patients' Voice and on a number of breast cancer clinical trials. The patient advocate is a member of the Breast Cancer Now tissue bank management board, the National Cancer Research Institute Breast Clinical Studies Group and is the lead patient representative for the National Institute for Health Research Cancer and Nutrition Collaboration. Additional areas of involvement include review of study documentation; attendance at trial steering group meetings and ongoing advice, guidance and patient perspective both at these meetings and as required on an ongoing basis; providing guidance on the use and content of study newsletters; and advice on materials and methods to disseminate the final study outcomes.

\section{ETHICS AND DISSEMINATION}

This is an observational cohort study and patient clinical management will not change as a result of the study. The study was approved on 30 January 2020 by the Hampshire B Research Ethics Committee (Level 3, Block B, Whitefriars, Lewins Mead, Bristol; Ref: 19/SC/0596. IRAS: 263666).

The study is listed on the ISRCTN registry.

The chief investigator and coinvestigators will be responsible for publication of the study findings in a peerreviewed scientific journal, on behalf of all collaborators. The manuscript will be prepared by a writing group, and participating investigators will be selected to join the writing group on the basis of contribution and following standard protocols for authorship.

Anonymous data will be available for request from 3 months after publication of the primary endpoint manuscript until the end of the archive and storage period. It will be available to researchers who provide a completed data sharing request form that describes a methodologically sound proposal, for the purpose of the approved proposal and if appropriate, signed a data sharing agreement. Proposals will be reviewed for approval by the study 
steering committee. Data for approved applications will be shared once all parties have signed relevant data sharing documentation, covering the study steering committee conditions for sharing and if required, an additional data sharing agreement from sponsor. Proposals should be directed to the chief investigator.

\section{Author affiliations}

${ }^{1}$ Cancer Sciences Academic Unit, University of Southampton, Southampton, UK ${ }^{2}$ Southampton Clinical Trials Unit, University of Southampton, Southampton, UK ${ }^{3}$ Clinical Informatics Research Unit, University of Southampton, Southampton, UK ${ }^{4}$ Human Development and Health Academic Unit, University of Southampton, Southampton, UK

${ }^{5}$ Independent Cancer Patients' Voice, London, UK

${ }^{6}$ University of Southampton, Faculty of Medicine, Southampton, UK

Acknowledgements The authors would like to thank the patients with breast cancer involved in the study, and the Southampton Breast Research Team, Clinical Trials Unit and Clinical Informatics Research Unit, University of Southampton.

Collaborators CAND0-3 steering group: James Batchelor; Ellen R Copson; Ramsey I Cutress; Kesta Durkin; Sean Ewings; Mike Grocott; Michelle Harvie; Adam Heetun; Sandy Jack; Alan Jackson; Fiona James; Richard Munday; Lesley Turner; Stephen Wootton.

Contributors RIC: chief investigator for study funding application; study conceptualisation, manuscript and protocol writing. ERC, SAW: coinvestigators for study funding application; study conceptualisation, manuscript and protocol writing. $\mathrm{KD}$ : manuscript and protocol writing; manuscript review and editing. $\mathrm{AH}$ : manuscript review. SE: power calculations, statistics and manuscript review. RM: study clinical informatics and manuscript review. LT: patient advocate, manuscript review. CANDO-3 Steering Group: manuscript review and approval.

Funding This study is funded by a grant from the World Cancer Research Fund International (reference: 2018/1807). The pilot work that informed the design of this work (CANDO-2 study) was funded by Breast Cancer Now (award ref: 2013NovSP227).

Competing interests SAW holds an investigator-led collaborative research agreement between Seca Medical Measuring Systems and Scales and the University of Southampton/University Hospital Southampton NHS Foundation Trust. Seca played no part in the design, conduct or interpretation of the work reported in this publication.

Patient and public involvement Patients and/or the public were involved in the design, or conduct, or reporting, or dissemination plans of this research. Refer to the Methods section for further details.

Patient consent for publication Not applicable.

Provenance and peer review Not commissioned; externally peer reviewed.

Open access This is an open access article distributed in accordance with the Creative Commons Attribution Non Commercial (CC BY-NC 4.0) license, which permits others to distribute, remix, adapt, build upon this work non-commercially, and license their derivative works on different terms, provided the original work is properly cited, appropriate credit is given, any changes made indicated, and the use is non-commercial. See: http://creativecommons.org/licenses/by-nc/4.0/.

ORCID iDs

Kesta Durkin http://orcid.org/0000-0002-5533-1662

Sean Ewings http://orcid.org/0000-0001-7214-4917

\section{REFERENCES}

1 World cancer research Fund/American Institute for cancer research. Physical activity, and the prevention of cancer: a global perspective. Washington DC: AICR, 2007.

2 Ligibel JA, Strickler HD. Obesity and its impact on breast cancer. Am Soc Clin Oncol Educ Book 2013;2013:52-9.

3 Cheraghi Z, Poorolajal J, Hashem T, et al. Effect of body mass index on breast cancer during premenopausal and postmenopausal periods: a meta-analysis. PLoS One 2012;7:e51446.

4 Renehan AG, Tyson M, Egger M, et al. Body-Mass index and incidence of cancer: a systematic review and meta-analysis of prospective observational studies. Lancet 2008;371:569-78.
5 Protani M, Coory M, Martin JH. Effect of obesity on survival of women with breast cancer: systematic review and meta-analysis. Breast Cancer Res Treat 2010;123:627-35.

6 Ewertz M, Jensen M-B, Gunnarsdóttir Katrín Á, et al. Effect of obesity on prognosis after early-stage breast cancer. J Clin Oncol 2011;29:25-31.

7 Copson ER, Cutress RI, Maishman T, et al. Obesity and the outcome of young breast cancer patients in the UK: the POSH study. Ann Oncol 2015;26:101-12.

8 Carmichael AR. Obesity and prognosis of breast cancer. Obes Rev 2006; 7:333-40

9 El-Tamer MB, Ward BM, Schifftner T, et al. Morbidity and mortality following breast cancer surgery in women: national benchmarks for standards of care. Ann Surg 2007;245:665-71.

10 Renehan AG, Harvie M, Cutress RI, et al. How to manage the obese patient with cancer. J Clin Oncol 2016;34:4284-94.

11 Carroll J, Protani M, Walpole E, et al. Effect of obesity on toxicity in women treated with adjuvant chemotherapy for early-stage breast cancer: a systematic review. Breast Cancer Res Treat 2012;136:323-30.

12 Furlanetto J, Eiermann W, Marmé F, et al. Higher rate of severe toxicities in obese patients receiving dose-dense (DD) chemotherapy according to unadjusted body surface area: results of the prospectively randomized gain study. Ann Oncol 2016;27:2053-9.

13 Griggs JJ, Mangu PB, Anderson H, et al. Appropriate chemotherapy dosing for obese adult patients with cancer: American Society of clinical oncology clinical practice guideline. J Clin Oncol 2012;30:1553-61.

14 Romero-Corral A, Somers VK, Sierra-Johnson J, et al. Accuracy of body mass index in diagnosing obesity in the adult general population. Int J Obes 2008;32:959-66.

15 Kazemi-Bajestani SMR, Mazurak VC, Baracos V. Computed tomography-defined muscle and fat wasting are associated with cancer clinical outcomes. Semin Cell Dev Biol 2016;54:2-10.

16 Aleixo GFP, Williams GR, Nyrop KA, et al. Muscle composition and outcomes in patients with breast cancer: meta-analysis and systematic review. Breast Cancer Res Treat 2019;177:569-79.

17 Morgan DJ, Bray KM. Lean body mass as a predictor of drug dosage. Implications for drug therapy. Clin Pharmacokinet 1994;26:292-307.

18 Prado CMM, Baracos VE, McCargar LJ, et al. Sarcopenia as a determinant of chemotherapy toxicity and time to tumor progression in metastatic breast cancer patients receiving capecitabine treatment. Clin Cancer Res 2009;15:2920-6.

19 Prado CMM, Antoun S, Sawyer MB, et al. Two faces of drug therapy in cancer: drug-related lean tissue loss and its adverse consequences to survival and toxicity. Curr Opin Clin Nutr Metab Care 2011;14:250-4.

20 Del Fabbro E, Parsons $\mathrm{H}$, Warneke $\mathrm{CL}$, et al. The relationship between body composition and response to neoadjuvant chemotherapy in women with operable breast cancer. Oncologist 2012;17:1240-5.

21 van den Berg MMGA, Kok DE, Posthuma L, et al. Body composition is associated with risk of toxicity-induced modifications of treatment in women with stage I-IIIB breast cancer receiving chemotherapy. Breast Cancer Res Treat 2019;173:475-81.

22 Prado CMM, Baracos VE, Xiao J, et al. The association between body composition and toxicities from the combination of Doxil and trabectedin in patients with advanced relapsed ovarian cancer. Appl Physiol Nutr Metab 2014;39:693-8.

23 Shachar SS, Deal AM, Weinberg M, et al. Body composition as a predictor of toxicity in patients receiving anthracycline and taxanebased chemotherapy for early-stage breast cancer. Clin Cancer Res 2017;23:3537-43.

24 NICE. National Institute of health and care excellence: breast cancer quality standard QS12, 2011. Available: https://www.nice.org. uk/guidance/qs12/resources/breast-cancer-pdf-2098481951941 [Accessed 17 Jul 2018]

25 Kilgour RD, Cardiff K, Rosenthall L, et al. Use of prediction equations to determine the accuracy of whole-body fat and fat-free mass and appendicular skeletal muscle mass measurements from a single abdominal image using computed tomography in advanced cancer patients. Appl Physiol Nutr Metab 2016;41:70-5.

26 Griggs JJ, Bohlke K, Balaban EP, et al. Appropriate systemic therapy dosing for obese adult patients with cancer: ASCO guideline update. $J$ Clin Oncol 2021;39:2037-48.

27 Lechi NH, Smethurst A, James F, et al. Body composition changes in breast cancer patients: the CANDO-2 study. European Journal of Surgical Oncology 2016;42:S23. 
28 Lee SY, Gallagher D. Assessment methods in human body composition. Curr Opin Clin Nutr Metab Care 2008;11:566-72.

29 Bosy-Westphal A, Schautz B, Later W, et al. What makes a BIA equation unique? validity of eight-electrode multifrequency BIA to estimate body composition in a healthy adult population. Eur $\mathrm{J}$ Clin Nutr 2013;67 Suppl 1:S14-21.

30 National Institutes of Health, National Cancer Institute. Common terminology criteria for adverse events (CTCAE) version 5.0, 2017.

31 S Peine, S.K, Carrero I, Brundert M. Generation of normal ranges for measures of body composition in adults based on bioelectrical impedance analysis using the SecA mBCA. International Journal of Body Composition Research 2013;11:67-76.

32 Aaronson NK, Ahmedzai S, Bergman B, et al. The European organization for research and treatment of cancer QLQ-C30: a quality-of-life instrument for use in international clinical trials in oncology. J Natl Cancer Inst 1993;85:365-76.

33 Craig CL, Marshall AL, Sjöström M, et al. International physical activity questionnaire: 12-country reliability and validity. Med Sci Sports Exerc 2003;35:1381-95.

34 Wilson M-MG, Thomas DR, Rubenstein LZ, et al. Appetite assessment: simple appetite questionnaire predicts weight loss in community-dwelling adults and nursing home residents. Am J Clin Nutr 2005;82:1074-81.

35 Bush K, Kivlahan DR, McDonell MB, et al. The audit alcohol consumption questions (AUDIT-C): an effective brief screening test for problem drinking. ambulatory care quality improvement project (ACQUIP). alcohol use disorders identification test. Arch Intern Med 1998;158:1789-95. 